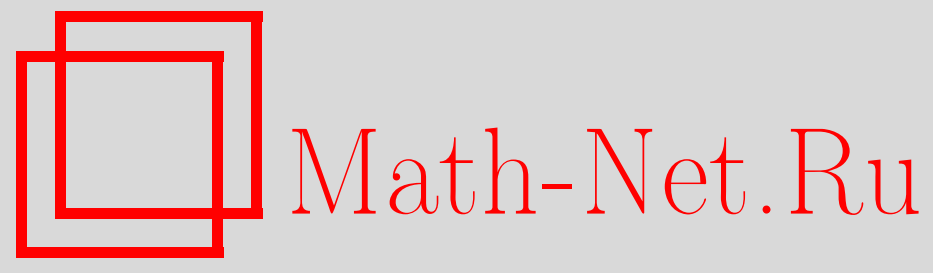

Ю. В. Немировский, А. И. Болтаев, Сложный изгиб и начальное разрушение гибридных деревянных брусьев, Вестн. Сам. гос. техн. ун-та. Сер. Физ.-мат. науки, 2017, номер 4, 699-716

DOI: https://doi.org/10.14498/vsgtu1572

Использование Общероссийского математического портала MathNet.Ru подразумевает, что вы прочитали и согласны с пользовательским соглашением

http://www . mathnet.ru/rus/agreement

Параметры загрузки:

IP: 18.207 .199 .55

26 апреля 2023 г., 15:22:35

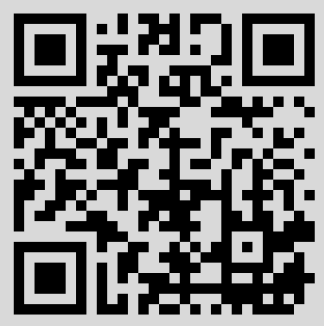


Вестн. Сам. гос. техн. ун-та. Сер. Физ.-мат. науки. 2017. Т. 21, № 4. С. $699-716$ ISSN: 2310-7081 (online), 1991-8615 (print)

УДК 539.3

\title{
Сложный изгиб и начальное разрушение гибридных деревянных брусьев
}

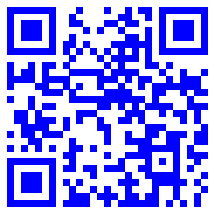

\author{
Ю. В. Немировский, А. И. Болтаев
}

Институт теоретической и прикладной механики им. С. А. Христиановича СО РАН, Россия, 630090, Новосибирск, ул. Институтская, 4/1.

\section{Аннотация}

Разработана математическая модель деформирования гибридных деревянных брусьев. Под гибридными понимаются брусья, образованные путем жесткого соединения (склеивания) по определенным контактным поверхностям набора слоев различных форм поперечных сечений и разных пород древесины. В общем случае брусья находятся в условиях сложного изгиба с растяжением-сжатием. Учитывается физическая нелинейность древесины, а также разное сопротивление растяжению и сжатию.

В общем случае задача сводится либо к решению системы трех нелинейных алгебраических уравнений третьей степени относительно обобщенных деформаций поперечного сечения либо к системе трех нелинейных обыкновенных дифференциальных уравнений относительно компонент вектора перемещения точек оси стержня. Для решения полученных алгебраических уравнений используется метод Ньютона, решение дифференциальных уравнений производится с помощью метода галеркинского типа.

Предложена аналитическая аппроксимация опытных диаграмм растяжения-сжатия древесины вдоль волокон в виде многочленов второй и третьей степени. Коэффициенты аппроксимирующих функций определяются двумя способами: с помощью метода наименьших квадратов, используя опытные диаграммы деформирования; с помощью наложения определенных требований на диаграммы, используя основные механические характеристики древесины (максимальные напряжения и деформации, модули упругости). Даны численные значения коэффициентов аппроксимации для 15-ти различных пород древесины.

Приведенные примеры расчетов гибридных деревянных брусьев показали возможность возникновения скрытых механизмов разрушения

\section{Научная статья}

(2)(7) Контент публикуется на условиях лицензии Creative Commons Attribution 4.0 International (https://creativecommons.org/licenses/by/4.0/deed.ru)

\section{Образец для цитирования}

Н ем и ро в с к и й Ю. В., Б олт а ев А. И. Сложный изгиб и начальное разрушение гибридных деревянных брусьев // Вестн. Сам. гос. техн. ун-та. Сер. Физ.-мат. науки, 2017. T. 21, № 4. C. 699-716. doi: 10.14498/vsgtu1572.

\section{Сведения об авторах}

Юрий Владимирович Немировский (D) http://orcid.org/0000-0002-4281-4358

доктор физико-математических наук, профессор; главный научный сотрудник; лаб. физики быстропротекающих процессов; e-mail: nemirov@itam.nsc.ru

Артем Иванович Болтаев (1) http://orcid.org/0000-0003-1317-9903

аспирант; старший лаборант; лаб. физики быстропротекающих процессов;

e-mail: boltaev_artem@mail.ru 
(когда предельные деформации достигаются во внутренних слоях стержня). Установлено существенное влияние перестановки материалов слоев на напряженно-деформированное состояние конструкции.

Разработанный в статье метод расчета гибридных стержневых деревянных конструкций открывает большие возможности для решения задач оптимизации при проектировании и позволяет рациональным способом использовать различные породы древесины.

Ключевые слова: слоистые конструкции, деревянные конструкции, диаграммы деформирования, физическая нелинейность, разносопротивляемость, сжатие, растяжение.

Получение: 18 октября 2017 г. / Исправление: 6 декабря 2017 г. / Принятие: 18 декабря 2017 г. / Публикация онлайн: 24 декабря 2017 г.

Введение. Древесина является одним из самых распространенных конструкционных материалов [1-3]. Она обладает такими качествами [1], как низкий удельный вес - при средней плотности $550 \mathrm{kг} / \mathrm{m}^{3}$ она в 14 раз легче стали и в 4.5 раза легче бетона; высокая удельная прочность $-25500 \mathrm{~m}^{2} / \mathrm{c}^{2}$ для древесины, $29500 \mathrm{~m}^{2} / \mathrm{c}^{2}$ для строительной стали и $5800 \mathrm{~m}^{2} / \mathrm{c}^{2}$ для бетона класса В25. К другим полезным качествам древесины относятся самовозобновляемость, экологичность, химическая стойкость, технологичность производства и др.

Существующие технологии производства деревянных конструкций способны создавать конструкции различных форм поперечных сечений и пролетов. При этом удается в значительной степени ослабить влияние основных недостатков древесины - неоднородность и анизотропию физико-механических свойств, различные пороки, пожароопасность, коробление, усушка и др. $[1,3]$.

Широкие возможности применения древесины в строительстве показывают такие сооружения, как радиобашня в Польше высотой 118 м (1935 г.), деревобетонный мост в Австрии [4] пролетом 85 м (1993 г.), жилое здание в Канаде [5] высотой 53 м (2017 г.) и др.

Сочетание таких материалов, как дерево, пластики, бетоны и металлы, позволяет создавать весьма эффективные конструкции [6, 7].

Используемые в проектной практике методы расчета деревянных конструкций $[8,9]$ значительно отстали от возможностей современного производства. Они в своей основе создавались до широкого внедрения компьютеров и в связи с этим обладают некоторыми недостатками:

- не учитывают реальные диаграммы деформирования древесины;

- не позволяют проектировать неоднородные (состоящие из разных пород) конструкции, так как основаны на экспериментальных данных, полученных для однородных конструкций;

- накладывают ограничения на возможные формы поперечных сечений (прямоугольное, круглое, двутавровое и т.п.).

Существующие методы расчета физически нелинейных стержневых конструкций [10-15] рассматривают в основном однородные конструкции и не уделяют должного внимания особенностям работы древесины.

На восполнение данных пробелов направлена настоящая работа. 
1. Диаграммы деформирования древесины. Здесь и далее будем говорить о диаграммах, полученных при кратковременных испытаниях малых чистых образцов древесины на растяжение-сжатие вдоль волокон.

Диаграммы деформирования древесины ели (линии 1) и лиственницы сибирской (линии 2) при растяжении (штрих-пунктирные линии) и сжатии (сплошные линии) вдоль волокон даны на рис. 1. Они построены по данным кратковременных испытаний малых чистых (без пороков) образцов [16-18]. Общим для данных диаграмм является то, что при растяжении древесина ведет себя практически линейно вплоть до разрушения, а при сжатии уже в области средних напряжений резко проявляет физическую нелинейность. Предел прочности на растяжение, как правило, больше предела прочности на сжатие. Данные особенности деформирования характерны и для других пород древесины.

Для использования диаграмм деформирования в расчетах необходимо получить их аналитическую

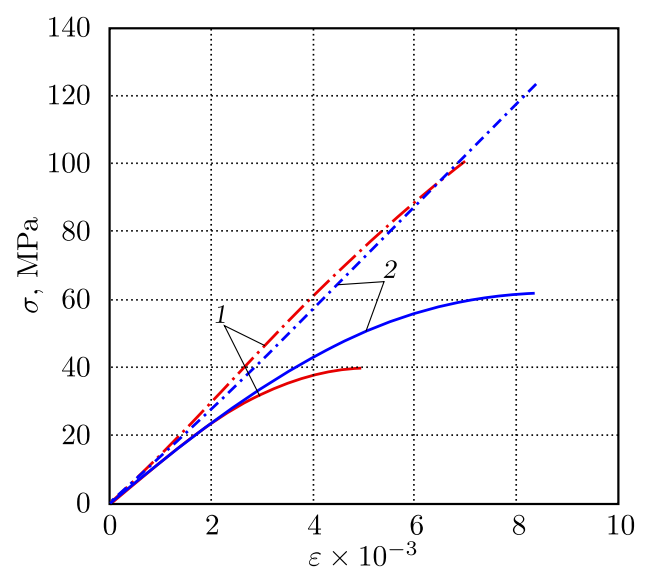

Рис. 1. Диаграммы деформирования древесины ели (линии 1) и лиственницы сибирской (линии 2) при растяжении (штрих-пунктирные линии) и сжатии (сплошные линии) вдоль волокон

[Figure 1. Timber deformation diagrams for Spruce (lines 1) and Siberian Larch (lines 2) under tension (dot-dashed lines) and compression (solid lines) along the fibers] форму. Функция, описывающая диаграммы, должна удовлетворять двум основным критериям: достаточно точно описывать опытные данные и иметь, по возможности, простой вид. При этом оба условия являются взаимоисключающими - желание получить как можно более точное совпадение с экспериментальными данными приводит к усложнению связи между напряжениями и деформациями.

Отметим, что не следует стремиться к идеальному совпадению экспериментальной диаграммы и аппроксимирующей ее функции, так как сами опытные диаграммы деформирования получаются путем осреднения целого набора диаграмм. При этом средние коэффициенты вариации механических свойств древесины: предел прочности, модуль упругости и др. лежат в пределах 13-20\% [19]. Для разработки теории расчета физически нелинейных гибридных стержневых систем будем использовать два вида функций:

1) квадратичная аппроксимация [20] отдельно для растяжения и сжатия

$$
\sigma^{ \pm}(\varepsilon)=E_{1}^{ \pm} \varepsilon+E_{2}^{ \pm} \varepsilon^{2}
$$

верхние знаки берутся при $0 \leqslant \varepsilon \leqslant \varepsilon_{*}^{+}$, нижние - при $\varepsilon_{*}^{-} \leqslant \varepsilon \leqslant 0$;

2) кубическая аппроксимация [21] на всем диапазоне деформирования $\varepsilon_{*}^{-} \leqslant \varepsilon \leqslant \varepsilon_{*}^{+}$

$$
\sigma(\varepsilon)=E_{1} \varepsilon+E_{2} \varepsilon^{2}+E_{3} \varepsilon^{3},
$$

где $\varepsilon_{*}^{+}, \varepsilon_{*}^{-}$- предельные значения продольных деформаций при растяжении $(+)$ и сжатии $(-) ; E_{i}^{ \pm}, E_{j}$ - коэффициенты аппроксимации диа- 
грамм деформирования соответственно для квадратной и кубической функций.

Принятие в качестве аппроксимирующей функции степенных многочленов, с одной стороны, позволяет достаточно точно описать экспериментальные данные; с другой стороны, данные функции имеют простой вид и являются одними из наиболее изученных в математике.

Связь между напряжениями и деформациями в форме (1) и (2) позволяет получать в пределе модели одномодульного и разномодульного линейноупругого материала. Если в (1) принять $E_{2}^{ \pm}=0$, то приходим к модели разномодульного линейно-упругого материала. Далее, принимая $E_{1}^{+}=E_{1}^{-}=E$, получим уравнение закона Гука. То же получим и в (2) при $E_{2}=E_{3}=0$.

В работе [22] была показана возможность аппроксимации диаграмм деформирования функциями (1) и $(2)$, а также приведены коэффициенты $E_{i}^{ \pm}$, $E_{j}$ для 15 различных пород древесины (см. табл. 1). В табл. $1 \sigma_{*}^{ \pm}$- предельные значения напряжений при растяжении $(+)$ и сжатии $(-)$.

Анализируя данные табл. 1, можно выявить следующие особенности диаграмм деформирования древесины:

1) различие в модулях упругости древесины при растяжении и сжатии $\left(E_{1}^{+}\right.$и $E_{1}^{-}$соответственно) для 12 из 15 пород составляет меньше $20 \%$, максимальное различие имеет место для сосны $-42.5 \%$;

2) предельные деформации растяжения практически всегда больше предельных деформаций сжатия; для березы даурской $\varepsilon_{*}^{+}$больше $\varepsilon_{*}^{-}$в 1.75 раза, а для граба кавказского $\varepsilon_{*}^{-}$больше $\varepsilon_{*}^{+}$в 1.45 раза;

3) предел прочности на растяжение больше предела прочности на сжатие в среднем в 2.5 раза; для березы даурской $\sigma_{*}^{+}$больше $\sigma_{*}^{-}$в 4.0 раза, для граба кавказского $\sigma_{*}^{+}$больше $\sigma_{*}^{-}$в 1.6 раза.

2. Напряженно-деформированное состояние бруса. Схема бруса в декартовой системе координат представляет собой стержень, состоящий из нескольких слоев. Слои могут быть выполнены из различных материалов и могут располагаться как горизонтально, так и вертикально (см. рис. 2). Общее число слоев принципиально не ограничено. Условия контакта слоев считаются совершенными - отсутствуют взаимные смещения любого направления в плоскости контакта.
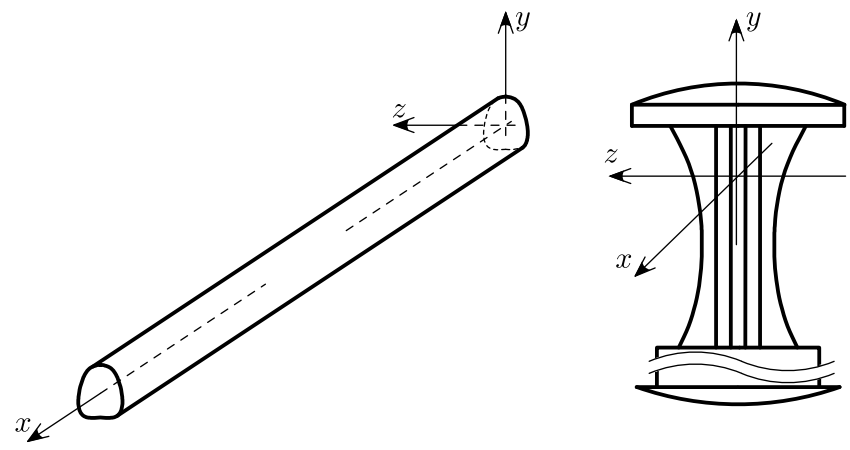

Рис. 2. Общий вид и форма поперечного сечения слоистого стержня

[Figure 2. General view and shape of the cross-section of the laminated rod] 
范

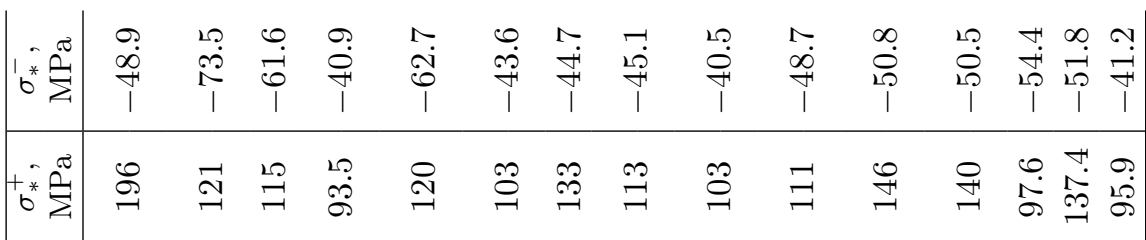

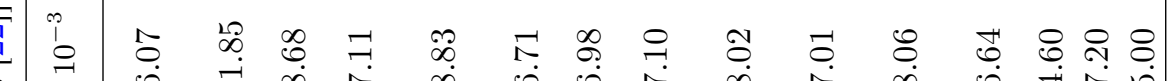

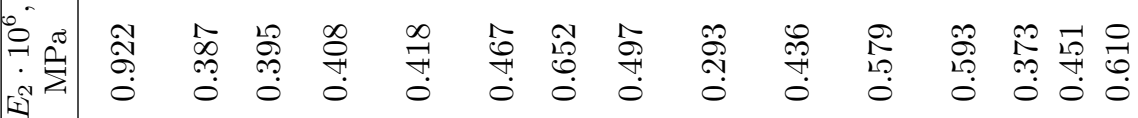

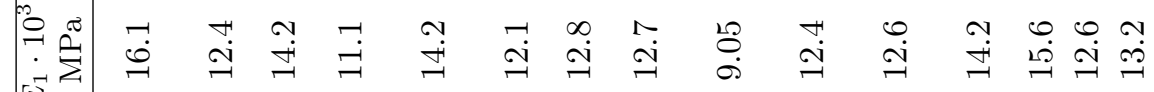
近

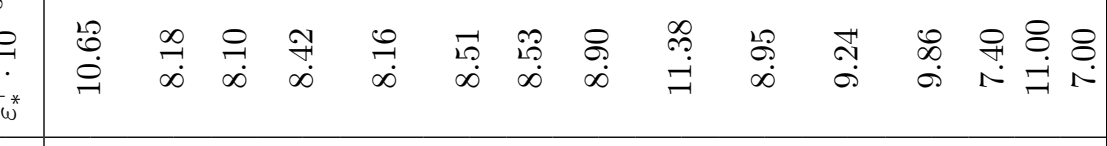

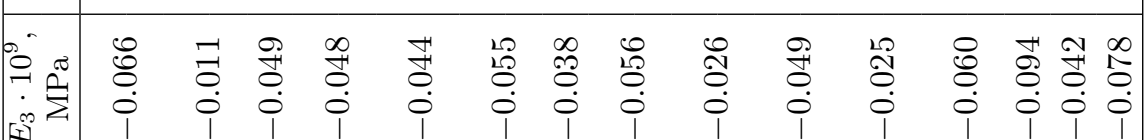
a 
Начало декартовой системы координат помещаем в левый конец стержня. Ось $x$ совпадает с продольной осью стержня и имеет определенную геометрическую привязку к поперечному сечению.

Для упрощения получающихся в дальнейшем решений будем считать, что структуры гибридных стержней и распределений внешних нагрузок согласованы так, что они в процессе нагружения либо вовсе не вызывают эффектов закручивания, либо они столь незначительны, что ими можно пренебречь. В общем случае стержень испытывает изгиб в плоскостях $x y$ и $y z$ с растяжением-сжатием. Все нагрузки приведены к продольной оси стержня - оси $x$.

Деформации и перемещения будем считать малыми. Это позволит записывать уравнения равновесия для недеформированного состояния:

$$
\frac{d^{2} M_{z}}{d x^{2}}=q_{y}-\frac{d m_{z}}{d x}, \quad \frac{d^{2} M_{y}}{d x^{2}}=q_{z}-\frac{d m_{y}}{d x}, \quad \frac{d N}{d x}=-q_{x} .
$$

Здесь $N$ - проекция вектора внутреннего усилия на ось $x, M_{z}, M_{y}$ - проекции вектора внутреннего момента на оси $z$ и $y$. Точка приложения вектора внутренних усилий лежит на оси стержня. Величины $q_{x}, q_{y}, q_{z}$ - проекции вектора распределенной нагрузки, приложенной к оси стрежня; $m_{z}, m_{y}$ - проекции вектора распределенного момента на оси $z$ и $y$.

Интегрируя уравнения (3), получим выражения для внутренних усилий:

$$
\begin{aligned}
& N(x)=N(0)-\int_{0}^{x} q_{x} d x \\
& M_{z}(x)=M_{z}(0)-Q_{y}(0) x-\int_{0}^{x} m_{z} d x+\int_{0}^{x}\left(\int_{0}^{x} q_{y} d x\right) d x, \\
& M_{y}(x)=M_{y}(0)-Q_{z}(0) x-\int_{0}^{x} m_{y} d x+\int_{0}^{x}\left(\int_{0}^{x} q_{z} d x\right) d x .
\end{aligned}
$$

В случае если стержень статически определимый, то значения внутренних усилий в начале координат определяем из условий равновесия узлов. Иначе необходимо составлять дополнительные условия совместности деформаций.

Принимается справедливой теория плоских сечений Бернулли и упрощенное выражение для кривизны плоской кривой. В соответствии с данными ограничениями связь между деформациями и перемещениями стержня выражается следующими известными соотношениями:

$$
\begin{aligned}
& \varepsilon(x, y, z)=\varepsilon_{0}-y \cdot \kappa_{z}-z \cdot \kappa_{y}, \\
& \varepsilon(x)=\frac{d u_{0}}{d x}, \quad \kappa_{y}=\frac{d^{2} v_{0}}{d x^{2}}, \quad \kappa_{z}=\frac{d^{2} w_{0}}{d x^{2}} .
\end{aligned}
$$

Здесь $\varepsilon_{0}, \kappa_{z}, \kappa_{y}$ - продольная деформация на уровне оси стержня и изменение кривизны оси стержня в проекции на оси $z$ и $y ; u_{0}(x), v_{0}(x), w_{0}(x)-$ компоненты перемещений точек на выбранной осевой линии. Интегрируя соотношения (5), получим

$$
\begin{aligned}
& u_{0}(x)=u_{0}(0)+\int_{0}^{x} \varepsilon_{0} d x \\
& v_{0}(x)=v_{0}(0)+\varphi_{y}(0) x+\int_{0}^{x}\left(\int_{0}^{x} \kappa_{y} d x\right) d x
\end{aligned}
$$




$$
\begin{aligned}
& \varphi_{y}(x)=\varphi_{y}(0)+\int_{0}^{x} \kappa_{y} d x \\
& w_{0}(x)=w_{0}(0)+\varphi_{z}(0) x+\int_{0}^{x}\left(\int_{0}^{x} \kappa_{z} d x\right) d x \\
& \varphi_{z}(x)=\varphi_{z}(0)+\int_{0}^{x} \kappa_{z} d x .
\end{aligned}
$$

Величины $u_{0}(0), v_{0}(0), w_{0}(0), \varphi_{y}(0)$ и $\varphi_{z}(0)$ находим из условий закрепления стержня.

Связь между нормальными напряжениями и деформациями $m$-того слоя выражается степенным многочленом вида (2):

$$
\sigma_{m}=\sum_{n=1}^{3} E_{n m} \varepsilon_{m}^{n}, \quad-\varepsilon_{m *}^{-} \leqslant \varepsilon_{m} \leqslant \varepsilon_{m *}^{+},
$$

где $E_{n m}$ - константы, зависящие от механических свойств $m$-того слоя стержня, $\varepsilon_{(m *)}^{ \pm}$- предельно допустимые продольные деформации $m$-того слоя при растяжении $(+)$ и сжатии $(-)$.

3. Система разрешающих уравнений. Вариант 1. Выразим интегральные внутренние силовые факторы $N, M_{y}$ и $M_{z}$ через продольные напряжения $\sigma_{m}$ :

$$
N=\sum_{m=1}^{n_{l}} \int_{F_{m}} \sigma_{m} d F_{m}, M_{y}=\sum_{m=1}^{n_{l}} \int_{F_{m}} \sigma_{m} z d F_{m}, M_{z}=\sum_{m=1}^{n_{l}} \int_{F_{m}} \sigma_{m} y d F_{m} .
$$

В (8) интегрирование ведется по площади поперечного сечения $m$-того слоя стержня. Количество слоев задается параметром $n_{l}$.

Подставляем в (8) связь между напряжениями и деформациями в виде (7) и первое уравнение из (5). После преобразований получим систему из трех нелинейных алгебраических уравнений 3-й степени относительно трех неизвестных $-\kappa_{y}, \kappa_{z}$ и $\varepsilon_{0}$ :

$$
\begin{array}{r}
\sum_{n=1}^{3} \sum_{k=1}^{n+1} \sum_{t=1}^{k}(-1)^{k-1} C_{n}^{k-1} C_{k-1}^{t-1} \varepsilon_{0}^{n-k+1} \kappa_{z}^{k-t} \kappa_{y}^{t-1} D_{n k t}-N= \\
=F_{1}\left(\varepsilon_{0}, \kappa_{y}, \kappa_{z}\right)=0 \\
\sum_{n=1}^{3} \sum_{k=1}^{n+1} \sum_{t=1}^{k}(-1)^{k-1} C_{n}^{k-1} C_{k-1}^{t-1} \varepsilon_{0}^{n-k+1} \kappa_{z}^{k-t} \kappa_{y}^{t-1} D_{n k(t+1)}+M_{y}= \\
=F_{2}\left(\varepsilon_{0}, \kappa_{y}, \kappa_{z}\right)=0 \\
\sum_{n=1}^{3} \sum_{k=1}^{n+1} \sum_{t=1}^{k}(-1)^{k-1} C_{n}^{k-1} C_{k-1}^{t-1} \varepsilon_{0}^{n-k+1} \kappa_{z}^{k-t} \kappa_{y}^{t-1} D_{n(k+1) t}+M_{z}= \\
=F_{3}\left(\varepsilon_{0}, \kappa_{y}, \kappa_{z}\right)=0
\end{array}
$$

где

$$
D_{n k t}=\sum_{m=1}^{n_{l}} E_{n m} d_{m k t}, \quad d_{m k t}=\int_{F_{m}} y^{k-t} z^{t-1} d F_{m}, \quad C_{n}^{k}=\frac{n !}{k !(n-k) !} .
$$


Система (9) обладает следующими характерными особенностями:

1) структура каждого уравнения одинаковая и отличается только коэффициентами $D_{n k t}$;

2) геометрия поперечного сечения полностью описывается коэффициентами $d_{m k t}$, характеристики диаграммы деформирования - коэффициентами $E_{n m}$; коэффициенты $D_{n k t}$ полностью включают в себя геометрические и механические характеристики слоев;

3) приняв $E_{n m}=0, n=2,3$, получим определяющие уравнения для слоистого линейно-упругого стержня; если при этом $n=1$, то приходим к уравнениям технической теории изгиба стержней [23].

Условие прочности задается соотношением

$$
\left|\varepsilon_{m, \max }^{ \pm}\right| \leqslant \varepsilon_{m *}^{ \pm}, \quad m=1,2, \ldots, n_{l},
$$

где $\varepsilon_{m, \max }^{ \pm}$- максимальные продольные деформации растяжения $(+)$и сжатия $(-) m$-того слоя. Проверка выполнения условия прочности осуществляется в каждом слое поперечного сечения. Так как функция продольных деформаций $\varepsilon(x, y, z)$ в пределах определенного поперечного сечения линейная (гипотеза плоских сечений), экстремальные значения она будет принимать на контуре поперечного сечения слоя. Задача определения области возможных значений $\kappa_{y}, \kappa_{z}$ и $\varepsilon_{0}$ представляет собой отдельный интерес. В случае прямого поперечного изгиба она сводится к решению задачи линейного программирования [24].

4. Решение системы разрешающих уравнений (9). Система нелинейных уравнений (9) такова, что можно легко получить аналитическое выражение производных входящих в нее функций по основным неизвестным $\kappa_{y}, \kappa_{z}$ и $\varepsilon_{0}$. Поэтому для ее решения будем использовать метод Ньютона [25]. Полагаем, что задача статически определима и, следовательно, внутренние усилия определены из (4).

Для произвольного поперечного сечения преобразуем систему (9) к векторному виду

$$
\bar{F}(\bar{x})=0 .
$$

Тогда решение строится в виде рекуррентной формулы

$$
\bar{x}^{j+1}=\bar{x}^{j}-J^{-1}\left(\bar{x}^{j}\right) \cdot \bar{F}\left(\bar{x}^{j}\right),
$$

где $J(\bar{x})$ - матрица Якоби системы $(11), \bar{x}^{j}$ - вектор неизвестных на $j$-том шаге. Каждый член $J(\bar{x})$ представляет собой нелинейный алгебраический многочлен:

$$
\begin{aligned}
& \frac{\partial F_{1}}{\partial \varepsilon_{0}}=\sum_{n=1}^{3} \sum_{k=1}^{n} \sum_{t=1}^{k}(-1)^{k-1}(n-k+1) C_{n}^{k-1} C_{k-1}^{t-1} \varepsilon_{0}^{n-k} \kappa_{z}^{k-t} \kappa_{y}^{t-1} D_{n k t}, \\
& \frac{\partial F_{1}}{\partial \kappa_{y}}=\sum_{n=1}^{3} \sum_{k=2}^{n+1} \sum_{t=2}^{k}(-1)^{k-1}(t-1) C_{n}^{k-1} C_{k-1}^{t-1} \varepsilon_{0}^{n-k+1} \kappa_{z}^{k-t} \kappa_{y}^{t-2} D_{n k t}, \\
& \frac{\partial F_{1}}{\partial \kappa_{z}}=\sum_{n=1}^{3} \sum_{k=2}^{n+1} \sum_{t=1}^{k-1}(-1)^{k-1}(k-t) C_{n}^{k-1} C_{k-1}^{t-1} \varepsilon_{0}^{n-k+1} \kappa_{z}^{k-t-1} \kappa_{y}^{t-1} D_{n k t},
\end{aligned}
$$




$$
\begin{aligned}
& \frac{\partial F_{2}}{\partial \varepsilon_{0}}=\sum_{n=1}^{3} \sum_{k=1}^{n} \sum_{t=1}^{k}(-1)^{k-1}(n-k+1) C_{n}^{k-1} C_{k-1}^{t-1} \varepsilon_{0}^{n-k} \kappa_{z}^{k-t} \kappa_{y}^{t-1} D_{n k(t+1)}, \\
& \frac{\partial F_{2}}{\partial \kappa_{y}}=\sum_{n=1}^{3} \sum_{k=2}^{n+1} \sum_{t=2}^{k}(-1)^{k-1}(t-1) C_{n}^{k-1} C_{k-1}^{t-1} \varepsilon_{0}^{n-k+1} \kappa_{z}^{k-t} \kappa_{y}^{t-2} D_{n k(t+1)}, \\
& \frac{\partial F_{2}}{\partial \kappa_{z}}=\sum_{n=1}^{3} \sum_{k=2}^{n+1}(-1)^{k-1}(k-t) C_{n}^{k-1} C_{k-1}^{t-1} \varepsilon_{0}^{n-k+1} \kappa_{z}^{k-t-1} \kappa_{y}^{t-1} D_{n k(t+1)}, \\
& \frac{\partial F_{3}}{\partial \varepsilon_{0}}=\sum_{n=1}^{3} \sum_{k=1}^{n} \sum_{t=1}^{k}(-1)^{k-1}(n-k+1) C_{n}^{k-1} C_{k-1}^{t-1} \varepsilon_{0}^{n-k} \kappa_{z}^{k-t} \kappa_{y}^{t-1} D_{n(k+1) t}, \\
& \frac{\partial F_{3}}{\partial \kappa_{y}}=\sum_{n=1}^{3} \sum_{k=2}^{n+1} \sum_{t=2}^{k}(-1)^{k-1}(t-1) C_{n}^{k-1} C_{k-1}^{t-1} \varepsilon_{0}^{n-k+1} \kappa_{z}^{k-t} \kappa_{y}^{t-2} D_{n(k+1) t}, \\
& \frac{\partial F_{3}}{\partial \kappa_{z}}=\sum_{n=1}^{3} \sum_{k=2}^{n+1} \sum_{t=1}^{k-1}(-1)^{k-1}(k-t) C_{n}^{k-1} C_{k-1}^{t-1} \varepsilon_{0}^{n-k+1} \kappa_{z}^{k-t-1} \kappa_{y}^{t-1} D_{n(k+1) t},
\end{aligned}
$$

Выражения частных производных $\frac{\partial F_{i}}{\partial \varepsilon_{0}}$ отличаются только коэффициентами $D_{n k t}$, как и выражения для $\frac{\partial F_{i}}{\partial \kappa_{y}}$, а также для $\frac{\partial F_{i}}{\partial \kappa_{z}}$. Необходимо отметить, что в (13) изменились пределы суммирования по сравнению с (9).

Начальное приближение $\bar{x}^{1}$ можно взять в виде нулевого вектора, что будет соответствовать недеформированному состоянию стержня. С другой стороны, его можно определить из решения системы (9) при $E_{n m}=0, n=2,3$, что равносильно переходу к физически линейной задаче. В этом случае система (9) переходит в систему трех линейных уравнений:

$$
\begin{aligned}
& \varepsilon_{0} D_{111}-\kappa_{y} D_{122}-\kappa_{z} D_{121}-N=0 \\
& \varepsilon_{0} D_{112}-\kappa_{y} D_{123}-\kappa_{z} D_{122}+M_{y}=0 \\
& \varepsilon_{0} D_{121}-\kappa_{y} D_{132}-\kappa_{z} D_{131}+M_{z}=0 .
\end{aligned}
$$

Выпишем решение системы (14):

$$
\bar{x}^{1}=A^{-1} \cdot \bar{B}
$$

где

$$
\bar{x}^{1}=\left(\begin{array}{c}
\varepsilon_{0} \\
\kappa_{y} \\
\kappa_{z}
\end{array}\right), \quad A=\left(\begin{array}{lll}
D_{111} & -D_{122} & -D_{121} \\
D_{112} & -D_{123} & -D_{122} \\
D_{121} & -D_{132} & -D_{131}
\end{array}\right), \quad B=\left(\begin{array}{c}
N \\
-M_{y} \\
-M_{z}
\end{array}\right) .
$$

Примем следующие критерии окончания итерационного процесса (12) [26]:

$$
\begin{aligned}
& \left\|\bar{F}\left(\bar{x}^{j}\right)-\bar{F}\left(\bar{x}^{j+1}\right)\right\| \leqslant \tau_{F}\left(1+\left\|\bar{F}\left(\bar{x}^{j+1}\right)\right\|\right), \\
& \left\|\bar{x}^{j}-\bar{x}^{j+1}\right\| \leqslant \sqrt{\tau_{F}}\left(1+\left\|\bar{x}^{j+1}\right\|\right) .
\end{aligned}
$$


Оба неравенства являются признаками близости последовательностей $\bar{F}\left(\bar{x}^{j}\right)$ и $\bar{x}^{j}$ к своим пределам. Параметр $\tau_{F}$ имеет смысл желаемой точности решения - чем он меньше, тем выше точность. Его величина задается непосредственно в расчете и может изменяться в зависимости от полученных результатов. В неравенствах (15) используется евклидова норма.

Область возможных значений вектора неизвестных $\bar{x}$ ограничена условием прочности (10).

5. Система разрешающих уравнений. Подставляем выражения внутренних усилий (9) в дифференциальные уравнения равновесия (3):

$$
\begin{aligned}
& \sum_{n=1}^{3} \sum_{k=1}^{n+1} \sum_{t=1}^{k} D_{n k t}^{\prime} \frac{d}{d x}\left(\varepsilon_{0}^{n-k+1} \kappa_{y}^{t-1} \kappa_{z}^{k-t}\right)+q_{x}=0, \\
& \sum_{n=1}^{3} \sum_{k=1}^{n+1} \sum_{t=1}^{k} D_{n k(t+1)}^{\prime} \frac{d^{2}}{d x^{2}}\left(\varepsilon_{0}^{n-k+1} \kappa_{y}^{t-1} \kappa_{z}^{k-t}\right)-\frac{d m_{y}}{d x}+q_{z}=0, \\
& \sum_{n=1}^{3} \sum_{k=1}^{n+1} \sum_{t=1}^{k} D_{n(k+1) t}^{\prime} \frac{d^{2}}{d x^{2}}\left(\varepsilon_{0}^{n-k+1} \kappa_{y}^{t-1} \kappa_{z}^{k-t}\right)-\frac{d m_{z}}{d x}+q_{y}=0 .
\end{aligned}
$$

где

$$
\begin{aligned}
& D_{n k t}^{\prime}=(-1)^{k-1} C_{n}^{k-1} C_{k-1}^{t-1} D_{n k t}, \quad D_{n k(t+1)}^{\prime}=(-1)^{k-1} C_{n}^{k-1} C_{k-1}^{t-1} D_{n k(t+1)}, \\
& D_{n(k+1) t}^{\prime}=(-1)^{k-1} C_{n}^{k-1} C_{k-1}^{t-1} D_{n(k+1) t} .
\end{aligned}
$$

Далее переходим от обобщенных деформаций $\varepsilon_{0}, \kappa_{y}$ и $\kappa_{z}$ к компонентам вектора перемещений точек оси стержня $u_{0}, v_{0}$ и $w_{0}$. Для этого подставляем (5) в (16):

$$
\begin{gathered}
\sum_{n=1}^{3} \sum_{k=1}^{n+1} \sum_{t=1}^{k} D_{n k t}^{\prime} \frac{d}{d x}\left(\left(\frac{d u_{0}}{d x}\right)^{n-k+1}\left(\frac{d^{2} v_{0}}{d x^{2}}\right)^{t-1}\left(\frac{d^{2} w_{0}}{d x^{2}}\right)^{k-t}\right)+q_{x}=0 \\
\sum_{n=1}^{3} \sum_{k=1}^{n+1} \sum_{t=1}^{k} D_{n k(t+1)}^{\prime} \frac{d^{2}}{d x^{2}}\left(\left(\frac{d u_{0}}{d x}\right)^{n-k+1}\left(\frac{d^{2} v_{0}}{d x^{2}}\right)^{t-1}\left(\frac{d^{2} w_{0}}{d x^{2}}\right)^{k-t}\right)- \\
\sum_{n=1}^{3} \sum_{k=1}^{n+1} \sum_{t=1}^{k} D_{n(k+1) t}^{\prime} \frac{d m_{y}}{d x^{2}}\left(\left(\frac{d u_{0}}{d x}\right)^{n-k+1}\left(\frac{d^{2} v_{0}}{d x^{2}}\right)^{t-1}\left(\frac{d^{2} w_{0}}{d x^{2}}\right)^{k-t}\right)- \\
-\frac{d m_{z}}{d x}+q_{y}=0
\end{gathered}
$$

Выражения (16), как и (17), являются системой из трех нелинейных обыкновенных дифференциальных уравнений. Вместе с граничными условиями они представляют собой краевую задачу, решение которой полностью определяет напряженно-деформированное состояние стержня. 
6. Решение системы разрешающих уравнений (17). Решение системы (17) в общем виде представляет определенные математические трудности и представляет собой отдельное исследование. Поэтому здесь рассмотрим решение одной частной задачи методом галеркинского типа [27].

Рассмотрим стержень с жестко закрепленными краями, тогда приближенное решение уравнений (17) можно представить в виде

$$
u_{0}(x)=a \sin ^{2} \frac{\pi x}{l}, \quad v_{0}(x)=b \sin ^{2} \frac{\pi x}{l}, \quad w_{0}(x)=c \sin ^{2} \frac{\pi x}{l},
$$

где $l$ - длина стержня; $a, b, c$-коэффициенты, подлежащие определению. Как несложно проверить, в таком виде решение точно удовлетворяет однородным краевым условиям:

$$
\begin{gathered}
u_{0}(0)=u_{0}(l)=v_{0}(0)=v_{0}(l)=w_{0}(0)=w_{0}(l)=0 \\
\left.\frac{d u_{0}}{d x}\right|_{x=0}=\left.\frac{d u_{0}}{d x}\right|_{x=l}=\left.\frac{d v_{0}}{d x}\right|_{x=0}=\left.\frac{d v_{0}}{d x}\right|_{x=l}=\left.\frac{d w_{0}}{d x}\right|_{x=0}=\left.\frac{d w_{0}}{d x}\right|_{x=l}=0 .
\end{gathered}
$$

Подставим (18) в (17) и проинтегрируем полученное по переменной $x$ в пределах длины стержня. После преобразований получим систему трех нелинейных уравнений относительно коэффициентов $a, b, c$ :

$$
\begin{aligned}
&-D_{111}^{\prime} \frac{\pi^{2}}{2 l} a+D_{311}^{\prime} \frac{3 \pi^{4}}{8 l^{3}} a^{3}-D_{331}^{\prime} \frac{\pi^{6}}{2 l^{5}} a c^{3}+D_{332}^{\prime} \frac{\pi^{6}}{2 l^{5}} a b c--D_{333}^{\prime} \frac{\pi^{6}}{2 l^{5}} a b^{2}+\int_{0}^{l} q_{x} \sin ^{2} \frac{\pi x}{l} d x=0 \\
& \frac{2 \pi^{4}}{l^{3}}\left(D_{122}^{\prime} b+D_{123}^{\prime} c\right)+\frac{\pi^{6}}{2 l^{5}} a^{2}\left(D_{322}^{\prime} b+D_{323}^{\prime} c\right)+ \\
&+\frac{6 \pi^{8}}{l^{7}} b^{2}\left(D_{345}^{\prime} b+D_{344}^{\prime} c\right)+ \frac{6 \pi^{8}}{l^{7}} c^{2}\left(D_{343}^{\prime} b+D_{342}^{\prime} c\right)- \\
&-\int_{0}^{l}\left(\frac{d m_{y}}{d x}-q_{z}\right) \sin ^{2} \frac{\pi x}{l} d x=0
\end{aligned}
$$

$$
\begin{aligned}
& \frac{2 \pi^{4}}{l^{3}}\left(D_{122}^{\prime} b+D_{123}^{\prime} c\right)+ \frac{\pi^{6}}{2 l^{5}} a^{2}\left(D_{322}^{\prime} b+D_{323}^{\prime} c\right)+ \\
&+\frac{6 \pi^{8}}{l^{7}} b^{2}\left(D_{345}^{\prime} b+D_{344}^{\prime} c\right)+\frac{6 \pi^{8}}{l^{7}} c^{2}\left(D_{343}^{\prime} b+D_{342}^{\prime} c\right)- \\
&-\int_{0}^{l}\left(\frac{d m_{z}}{d x}-q_{y}\right) \sin ^{2} \frac{\pi x}{l} d x=0 .
\end{aligned}
$$

Система (19) решается методом Ньютона по формуле, аналогичной формуле (12).

7. Результаты расчетов. Рассмотрим балку длиной $l=600$ см с прямоугольным поперечным сечением $b \times h=20 \times 40$ см, которая имеет три слоя высотой $h_{1}, h_{2}$ и $h_{3}$, изготовленных из различных материалов. Расчетная схема балки приведена на рис. 3. Балка нагружена равномерно распределенной нагрузкой интенсивностью $q$. 

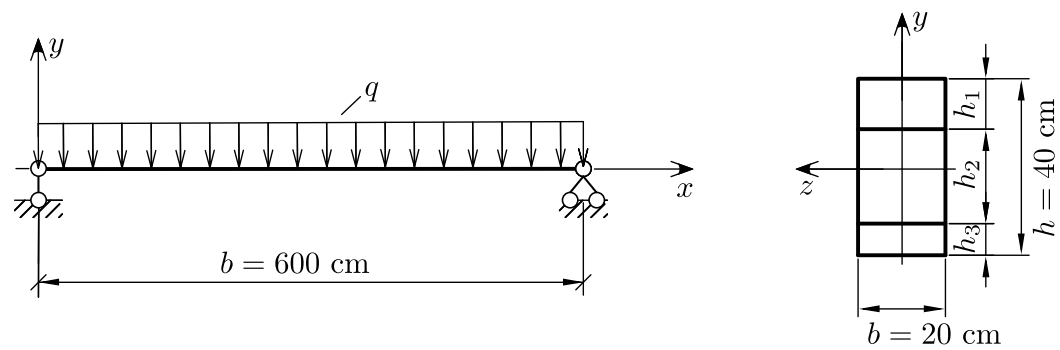

Рис. 3. Расчетная схема балки [Figure 3. The calculation scheme of a beam]

Расчеты будем производить по аналогии с работой [22]. В первой серии расчетов будем менять размеры и породы слоев. В каждом расчете будем нагружать конструкцию до тех пор, пока в каком либо слое максимальные деформации не достигнут предельных значений. Определение напряженнодеформированного состояния балки осуществлялось путем решения разрешающей системы уравнений (9) методом Ньютона. Определив обобщенные деформации поперечных сечений балки, перемещения точек оси находим по формулам (6), а продольные напряжения в слоях - по (7).

При данных условиях закрепления и характере нагрузки опасное сечение будет находиться в середине пролета. Волокна нижней части балки будут растянуты, верхней - сжаты.

Для выявления особенностей работы слоистой балки из разносопротивляющегося материала были проведены следующие расчеты. В первом расчете (строка 1 табл. 2) для всех слоев были взяты параметры сосны (333). Во втором - для верхнего слоя были взяты параметры породы, которая хорошо

Таблица 2

Результаты расчета однопролетной балки (см. рис. 3) [Results of the calculation of a single-span beam (see Fig. 3)]

\begin{tabular}{c|c|c|c|c|c|c|c|c|c}
\hline nos. & Timber & $q_{\max }, \mathrm{kN} / \mathrm{m}^{2}$ & $w_{\max }, \mathrm{cm}$ & $h_{1}, \mathrm{~cm}$ & $h_{2}, \mathrm{~cm}$ & $h_{3}, \mathrm{~cm}$ & $n^{*}$ & $W, \mathrm{kN}$ & $D$ \\
\hline 1 & 2 & 3 & 4 & 5 & 6 & 7 & 8 & 9 & 10 \\
\hline 1 & 333 & 79 & 7.7 & 13.3 & 13.3 & 13.3 & 1 & 2.40 & 0.53 \\
2 & $6-5$ & 108 & 12.9 & 20.0 & 0.0 & 20.0 & 1 & 3.08 & 0.56 \\
3 & $5-6$ & 76 & 9.2 & 20.0 & 0.0 & 20.0 & 1 & 3.08 & 0.58 \\
4 & $1-1$ & 65 & 8.3 & 20.0 & 0.0 & 20.0 & 1 & 2.12 & 0.78 \\
5 & 615 & 86 & 10.4 & 13.3 & 13.3 & 13.3 & 2 & 2.36 & 0.60 \\
\hline
\end{tabular}

Timber - распределение пород древесины в слоях балки [timber distribution in the layers of the beam]

$q_{\max }$ - предельно допустимая величина интенсивности нагрузки $q$ [the maximum allowable value of the load intensity $q$ ]

$w_{\max }$ - максимальный прогиб балки [the maximum deflection of the beam]

$h_{i}$ - толщина $i$-того слоя балки [the thickness of the $i$-th layer of the beam]

$n^{*}$ - номер слоя, в котором достигнуты предельные деформации [the layer number in which the limiting strains are reached]

$W$ - вес балки [the weight of beam]

$D$ - степень нагружения нижних волокон балки (см. (20)) [degree of loading of lower fibers of the beam (see Eq. (20))] 
сопротивляется сжатию (6), а для нижнего слоя - породы, хорошо сопротивляющейся растяжению (5). В третьем расчете слои из второго расчета менялись местами. В четвертом расчете для верхнего слоя брались параметры породы, плохо работающей на сжатие (1), а для нижнего слоя - породы, плохо работающей на растяжение (1). В пятом расчете для среднего слоя брались параметры одной из наиболее слабых пород (1). Результаты расчетов приведены в табл. 2.

В столбце 2 табл. 2 дано распределение пород по слоям. Каждая цифра характеризует породу определенного слоя (см. табл. 1). Так, шифр 615 означает, что в расчетах для первого слоя брались параметры лиственницы, для второго - параметры ели и для третьего - параметры березы. Цифрой 3 обозначены параметры сосны.

В столбцах 2 и 3 приведены максимальная нагрузка и максимальный прогиб балки. В столбце 10 приведена степень нагружения крайних нижних волокон:

$$
D=\max (\varepsilon(x,-0.5 h)) / \varepsilon_{3 *}^{+} .
$$

Результаты анализа выполненных расчетов позволяют сделать некоторые выводы:

1) варьирование пород слоев приводит к значительному изменению величины предельной нагрузки и максимального прогиба; предельная нагрузка изменяется в 1.66 раза, максимальный прогиб в 1.55 раза;

2) в расчете 5 (строка 5 табл. 2) предельные деформации были достигнуты во внутреннем слое балки, тогда как в однородных конструкциях предельные деформации всегда достигаются на фибровых волокнах балки;

3) в расчетах 1-4 предельные деформации достигались на верхних фибровых волокнах;

4) во всех расчетах нижние волокна балки были сильно недогружены, что является следствием значительной разносопротивляемости древесины.

Заключение. В работе представлено решение проблемы определения напряженно-деформированного состояния гибридных деревянных брусьев при сложном изгибе в условиях «растяжение-сжатие» с использованием аналитической аппроксимации диаграмм деформирования древесины полиномами 2-й и 3-й степени, которая хорошо согласуется с экспериментальными данными. Учитывается физическая нелинейность и разная сопротивляемость материала растяжению и сжатию. Примеры расчетов показывают следующие особенности деформирования гибридных деревянных брусьев:

1) в зависимости от распределения пород в сечении бруса возможно возникновение скрытых механизмов разрушения, когда предельное состояние достигается во внутренних слоях стержня;

2) простая перестановка слоев приводит к значительному изменению предельной нагрузки и максимальных перемещений, а также характера начала разрушения;

3) слоистое расположение материалов с различными физико-механическими свойствами в брусе открывает большие возможности для решения задач оптимизации. 
Конкурирующие интересы. Заявляем, что в отношении авторства и публикации этой статьи конфликта интересов не имеем.

Авторский вклад и ответственность. Все авторы принимали участие в разработке концепции статьи и в написании рукописи. Авторы несут полную ответственность за предоставление окончательной рукописи в печать. Окончательная версия рукописи была одобрена всеми авторами.

Финансирование. Работа выполнена в рамках гранта РФФИ № 15-01-00825_а.

\section{Библиографический список}

1. Арленинов Д. К., Буслаев Ю. Н., Игнатьев В. П., Романов П. Г., Чахов Д. К. Конструкиии из дерева и пластмасс. М.: АСB, 2002. 280 с.

2. Шмидт А. Б., Дмитриев А. П. Атлас строительных конструкций из клееной древесины и водостойкой фанеры. М.: АCB, 2002. 292 с.

3. Porteous J., Kermani A. Structural timber design to Eurocode 5. United Kingdom: John Wiley \& Sons, 2013. xii+542 pp. doi: 10.1002/9780470697818.

4. Pischl R., Schickhofer G. The Mur River wooden bridge, Austria// Structural Engineering International, 1993. vol. 3, no. 4. pp. 217-219. doi: 10.2749/101686693780607660.

5. Poirier E., Moudgil M., Fallahi A., Staub-French S., Tannert T. Design and construction of a 53-meter-tall timber building at the university of British Columbia/ Proc. of WCTE'22016 (Vienna, Austria, August 22-25, 2016), 2016. 10 pp., Retrieved from http://www.proholz. at/fileadmin/proholz/media/documents/Thomas-Tannert.pdf (December 06, 2017).

6. Стоянов В. В., Окунь И. В. Усиление балочных конструкций методом послойного армирования // Изв. вузов. Строительство, 2013. №11-12. С. 44-47.

7. Немировский Ю. В., Болтаев А. И. Особенности расчета деревожелезобетонного балочного моста // Вестник СибАДИ, 2016. №5. С. 114-124.

8. Коченов В. М. Несущая способность элементов и соединений деревянных конструкиий. М.: Госстройиздат, 1953. 320 с.

9. Деревянные конструкиии: СП 64.13330.2011. Свод правил. Актуализированная редакция СНиП ІІ-25-80. Введ. 20.05.2011. М., 2011. 92 с.

10. Ржаницын А. Р. Изгиб и сложное сопротивление прямоугольного сечения стержня при произвольной диаграмме работы материала / Расчет тонкостенных пространственных конструкиий: Сб. статей под ред. А. Р. Ржаницына. М., 1964. С. 7-22.

11. Геммерлинг А. В. Расчет стержневых систем. М.: Стройиздат, 1974. 208 с.

12. Лукаш А. П. Основы нелинейной строительной механики. М.: Стройиздат, 1978. 204 с.

13. Шапиро Д. М., Агарков А. В., Мельничук Н. Н., Чан Тхи Тхюи Ван Нелинейные методы расчета в современном проектировании // Научный журнал строительства и архитектурь, 2009. № 3. С. 85-94.

14. Owen D. R., Hinton E. Finite elements in plasticity: Theory and Practice. Swansea: John Wiley \& Sons, 2013. 640 pp.

15. McGuire W., Gallagher R. H., Ziemian R. D. Matrix structural analysis. New York: John Wiley \& Sons, 2014. xvii +460 pp.

16. Исследование прочности и деформативности древесины: Сб. статей / ред. Г. Г. Карлсен. М.: Госстройиздат, 1956. 172 с.

17. Быков В. В. Экспериментальные исследования прочности и деформативности древесины сибирской лиственницы при сжатии и растяжении вдоль волокон с учетом длительного действия нагрузки // Изв. вузов. Строительство, 1967. № 8. С. 3-8.

18. Квасников Е. Н. Вопросы длительного сопротивления древесины. Л.: Стройиздат, 1972. $96 \mathrm{c.}$

19. Боровиков А. М., Чибисова Г. А., Кот Л. В. Древесина. Показатели физико-механических свойств малых чистых образиов: ГСССД 69-84. Таблицы стандартных справочных данных. Введ. 01.06.1985. Минск: Госстандарт СССР, 1985. 29 с. 
20. Немировский Ю. В. Расчет и рациональное проектирование деревянных стержневых элементов/ Современные проблемы совершенствования и развития конструкиий в строительстве и транспорте: Сб. научных трудов III Междунар. научно-технич. конференции. Самара: СамГАСУ, 2005. С. 247-251.

21. Немировский Ю. В. Метод расчета композитных стрежневых систем из разномодульных материалов / Фундаментальные и прикладные проблемы современной механики: Материалы V Всероссийской научной конференции. Томск, 2006. С. 288-290.

22. Немировский Ю. В., Болтаев А. И. Влияние формы и расположения слоев на напряженно-деформированное состояние гибридных деревянных балок // Becmник БГТУ им. В. Г. Шухова, 2017. Т. 2, №10. С. 73-83. doi:10.12737/article 59cd0c5b5b1678.28291672.

23. Филин А. П. Прикладная механика твердого деформируемого тела. Т. 2. М.: Наука, 1978. $616 \mathrm{c.}$

24. Немировский Ю. В., Болтаев А. И. Особенности деформирования и разрушения деревянных клееных многопролетных балок. Сообщение $1 / /$ Изв. вузов. Строительство, 2016. №6. C. 116-126.

25. Ortega J. M., Rheinboldt W. C. Chapter 7 - General Iterative Methods / Iterative solution of nonlinear equations in several variables. New York: Academic Press, 1970. pp. 181-239. doi : 10.1016/B978-0-12-528550-6.50018-1.

26. Gill P. E., Murray W., Wright M. H. 8.2.3. Termination Criteria/ Practical optimization. London: Academic Press, 1981. pp. 305-310.

27. Fletcher C. A. J. Computational Galerkin Methods / Computational Galerkin Methods / Springer Series in Computational Physics. Berlin, Heidelberg: Springer, 1984. pp. 72-85. doi : 10.1007/978-3-642-85949-6_2. 


\title{
MSC: $74 C 99$
}

\section{Complex bending and initial destruction of hybrid timber beams}

\author{
Yu. V. Nemirovsky, A. I. Boltaev \\ Khristianovich Institute of Theoretical and Applied Mechanics, \\ Siberian Branch of the Russian Academy of Sciences, \\ 4/1, Institutskaya st., Novosibirsk, 630090, Russian Federation.
}

\begin{abstract}
A mathematical model of the deformation of hybrid timber beams has been developed. By hybrid we mean bars, formed by rigid connection (gluing) on certain contact surfaces of a set of layers of different forms of crosssections and different types of timber. In general, the bars are in conditions of complex bending with stretching-compression. The physical non-linearity of timber, as well as the different tensile and compression resistance, is taken into account.

In the general case, the problem reduces either to solving a system of three nonlinear algebraic equations of the third degree with respect to generalized deformations of the cross section or to a system of three nonlinear ordinary differential equations with respect to the components of the displacement vector of the points of the axis of the rod. To solve the obtained algebraic equations the Newton method is used, the solution of the differential equations is performed using the Galerkin type method.

An analytical approximation of the experimental tension-compression diagrams of timber along the fibers in the form of polynomials of the second and third degree is proposed. The coefficients of the approximating functions are determined in two ways: using the least squares method with the experimental deformation diagrams; by imposing certain requirements on the diagrams, using the basic mechanical characteristics of the timber (maximum stresses and deformations, moduli of elasticity). Numerical values of the approximation coefficients for 15 different types of timber are given.

The above examples of calculations of hybrid timber beams have shown the possibility of the emergence of hidden mechanisms of destruction, as well as the strong influence of the rearrangement of layer materials on the stress-strain state of the structure.
\end{abstract}

\section{Research Article}

(우(-) The content is published under the terms of the Creative Commons Attribution 4.0 International License (http://creativecommons.org/licenses/by/4.0/)

Please cite this article in press as:

Nemirovsky Yu. V., Boltaev A. I. Complex bending and initial destruction of hybrid timber beams, Vestn. Samar. Gos. Tekhn. Univ., Ser. Fiz.-Mat. Nauki [J. Samara State Tech. Univ., Ser. Phys. Math. Sci.], 2017, vol. 21, no. 4, pp. 699-716. doi: 10.14498/vsgtu1572 (In Russian).

\section{Authors' Details:}

Yuriy V. Nemirovsky (1) http://orcid.org/0000-0002-4281-4358

Dr. Phys. \& Math. Sci., Professor; Chief Researcher; Lab. of Fast Processes Physics; e-mail: nemirov@itam.nsc.ru 
The method developed in the article for the calculation of hybrid rodshaped timber structures offers great opportunities for solving optimization problems in the design, and allows rational use of various types of timber.

Keywords: layered structures, timber structures, deformation diagrams, physical nonlinearity, different resistance, compression, stretching.

Received: $18^{\text {th }}$ October, $2017 /$ Revised: $6^{\text {th }}$ December, $2017 /$ Accepted: $18^{\text {th }}$ December, $2017 /$ First online: $24^{\text {th }}$ December, 2017

Competing interests. We declare that we have no conflicts of interests with the authorship and publication of this article.

Authors' contributions and responsibilities. Each author has participated in the article concept development and in the manuscript writing. The authors are absolutely responsible for submitting the final manuscript in print. Each author has approved the final version of manuscript.

Funding. The work was carried out within the framework of the RFBR grant no. 1501-00825_a.

\section{References}

1. Arleninov D. K., Buslaev Yu. N., Ignat'ev V. P., Romanov P. G., Chakhov D. K. Konstruktsii iz dereva i plastmass [Construction of wood and plastics]. Moscow, ASV Publ., 2002, 280 pp. (In Russian)

2. Shmidt A. B., Dmitriev P. A. Atlas stroitel'nykh konstruktsii iz kleenoi drevesiny i vodostoikoi fanery [Atlas of building structures made of laminated wood and waterproof plywood]. Moscow, ASV Publ., 2002, 292 pp. (In Russian)

3. Porteous J., Kermani A. Structural timber design to Eurocode 5. United Kingdom, John Wiley \& Sons, 2013, xii+542 pp. doi : 10.1002/9780470697818.

4. Pischl R., Schickhofer G. The Mur River wooden bridge, Austria, Structural Engineering International, 1993, vol. 3, no. 4, pp. 217-219. doi: 10.2749/101686693780607660.

5. Poirier E., Moudgil M., Fallahi A., Staub-French S., Tannert T. Design and construction of a 53-meter-tall timber building at the university of British Columbia, In: Proc. of WCTE'22016 (Vienna, Austria, August 22-25, 2016), 2016, 10 pp., Retrieved from http:// www.proholz.at/fileadmin/proholz/media/documents/Thomas-Tannert.pdf (December 06, 2017).

6. Stoyanov V. V., Okun I. V. Strengthening of frame designs by the method of layer-by-layer reinforcing, Izv. vuzov. Stroitel'stvo, 2013, no. 11-12, pp. 44-47 (In Russian).

7. Nemirovsky Yu. V., Boltaev A. I. Calculation of wood reinforced concrete girder bridge, Vestnik SibADI, 2016, no. 5, pp. 114-124 (In Russian).

8. Kochenov V. M. Nesushchaia sposobnost' elementov $i$ soedinenii dereviannykh konstruktsii [Durability of Joining Elements of Timber Structures]. M., Gosstroiizdat, 1953, 320 pp. (In Russian)

9. Timber structures, SP 64.13330.2011. Set of Rules. Updated edition of SNiP II-25-80. Moscow, 2011, 92 pp. (In Russian)

10. Rzhanitsyn A. R. Bending and complex resistance of the rectangular cross-section of the rod for an arbitrary diagram of the work of the material, In: Raschet tonkostennykh prostranstvennykh konstruktsii [Calculation of thin-walled spatial structures], Collection of scientific works; ed. A. R. Rzhanitsyn. Moscow, 1964, pp. 7-22 (In Russian).

11. Gemmerling A. V. Raschet sterzhnevykh sistem [Calculation of rod systems]. Moscow, Stroiizdat, 1974, 208 pp. (In Russian)

12. Lukash A. P. Osnovy nelineinoi stroitel'noi mekhaniki [Fundamentals of nonlinear construction mechanics]. Moscow, Stroiizdat, 1978, 204 pp. (In Russian) 
13. Shapiro D. M., Agarkov A. V., Melnichuk N. N., Chan Tkhi Tkhui Van The non-linear methods of analysis in modern designing (by the example of geotechnics facilities and bridges), Russian Journal of Building Construction and Architecture, 2010, no. 3, pp. 46-58.

14. Owen D. R., Hinton E. Finite elements in plasticity: Theory and Practice. Swansea, John Wiley \& Sons, 2013, 640 pp.

15. McGuire W., Gallagher R. H., Ziemian R. D. Matrix structural analysis. New York, John Wiley \& Sons, 2014, xvii +460 pp.

16. Issledovanie prochnosti $i$ deformativnosti drevesiny [Investigation of strength and deformability of timber], Collection of scientific works, ed. G. G. Karlsen. Moscow, Gosstroiizdat, 1956, 172 pp. (In Russian)

17. Bykov V. V. Experimental studies of the strength and deformability of Siberian larch timber during compression and stretching along the fibers, taking into account the long-term action of the load, Izv. vuzov. Stroitel'stvo, 1967, no. 8, pp. 3-8 (In Russian).

18. Kvasnikov E. N. Voprosy dlitel'nogo soprotivleniia drevesiny [Problems of long-term resistance of timber]. Leningrad, Stroiizdat, 1972, 96 pp. (In Russian)

19. Borovikov A. M., Chibisova G. A., Kot L. V. Wood. Characteristics of physical-mechanical properties of small clear specimens, GSSSD 69-84. Tables of standard reference data. Minsk, State Standard of the USSR, 1985, 29 pp. (In Russian)

20. Nemirovsky Yu. V. Calculation and rational design of timber rod elements, In: Modern problems of improvement and development of designs in construction and transport, Proc. of the III International Scientific and Technical Conference. Samara, Samara State University of Architecture and Civil Engineering, 2005, pp. 247-251 (In Russian).

21. Nemirovsky Yu. V. The method of calculation of composite beam systems made of materials multimodulus, In: Fundamental and applied problems of modern mechanics, Proc. of the V All-Russian Scientific Conference. Tomsk, 2006, pp. 288-290 (In Russian).

22. Nemrovsky Yu. V., Boltaev A. I. Influence of the form and layout of layers on the stresseddeformed state of hybrid wooden beams, Bulletin of Belgorod State Technological University named after V. G. Shukhov, 2017, vol. 2, no. 10, pp. 73-83 (In Russian). doi: 10.12737/ article_59cd0c5b5b1678.28291672.

23. Filin A. P. Prikladnaya mekhanika tverdogo deformiruemogo tela [Applied Mechanics of solid deformable body], vol. 2. Moscow, Nauka, 1978, 616 pp. (In Russian)

24. Nemirovsky Yu. V., Boltaev A. I. Features of deformation and fracture of multi-span glued wooden beams. Message 1, Izv. vuzov. Stroitel'stvo, 2016, no. 6, pp. 116-126 (In Russian).

25. Ortega J. M., Rheinboldt W. C. Chapter 7 - General Iterative Methods, In: Iterative solution of nonlinear equations in several variables. New York, Academic Press, 1970, pp. 181-239. doi : 10.1016/B978-0-12-528550-6.50018-1.

26. Gill P. E., Murray W., Wright M. H. 8.2.3. Termination Criteria, In: Practical optimization. London, Academic Press, 1981, pp. 305-310.

27. Fletcher C. A. J. Computational Galerkin Methods, In: Computational Galerkin Methods, Springer Series in Computational Physics. Berlin, Heidelberg, Springer, 1984, pp. 72-85. doi: 10.1007/978-3-642-85949-6_2. 